\title{
ON GROUPS SATISFYING THE MAXIMAL AND THE MINIMAL CONDITIONS FOR SUBNORMAL SUBGROUPS OF INFINITE ORDER OR INDEX
}

\author{
Alessio Russo
}

\begin{abstract}
In this article we will prove that a generalized radical group satisfying the maximal condition for subnormal subgroups of infinite order (the minimal condition for subnormal subgroups of infinite index, respectively) is soluble-by-finite. Such result generalizes that obtained by D. H. Paek in [5].
\end{abstract}

\section{Introduction}

The maximal and the minimal conditions on systems of subgroups are some of the most important finiteness conditions in the theory of groups. The structure of (generalized) soluble groups satisfying the maximal or the minimal condition on all subgroups has completely been described, and there are also in the literature many results concerning groups with similar restrictions imposed only to certain relevant systems of subgroups, like those of abelian, normal or subnormal subgroups (see for instance [9], Chapter 3 and Chapter 5). Weaker forms of these chain conditions have also been considered; for instance, if $\chi$ is a property pertaining to subgroups, a group $G$ is said to satisfy the weak maximal condition (the weak minimal condition, respectively) on $\chi$-subgroups if, given an ascending (a descending, respectively) chain $X_{1}<X_{2}<\cdots$ (respectively, $X_{1}>X_{2}>\cdots$ ) of $\chi$-subgroups of $G$, only a finite number of the indices $\left|X_{\alpha+1}: X_{\alpha}\right|$ (respectively, $\left|X_{\alpha}: X_{\alpha+1}\right|$ ) are infinite. It was proved by Baer [1] and Zaičev [11], [12] that for a soluble group $G$ the weak maximal and the weak minimal conditions on all subgroups coincide, and are equivalent to the requirement that $G$ is a minimax group, i.e., a group having a finite series whose factors either satisfy the maximal or the minimal condition on subgroups. The structure of groups satisfying the weak chain conditions on normal or subnormal subgroups was later investigated by Kurdachenko [3], [4].

Received January 27, 2009.

2000 Mathematics Subject Classification. Primary 20E15; Secondary 20F22.

Key words and phrases. subnormal, maximal condition, minimal condition, soluble, subsoluble, radical. 
Let $\chi$ be a subgroup theoretical property. A group $G$ is said to satisfy the condition $\max -\infty \chi$ if there are no infinite strictly ascending chains of infinite $\chi$-subgroups of $G$; similarly, we shall say that $G$ satisfies min- $\infty \chi$ if there are no infinite strictly descending chains of $\chi$-subgroups of infinite index of $G$. Clearly, max- $\infty \chi$ implies the weak maximal condition on $\chi$-subgroups, with a similar statement for min- $\infty \chi$. The structure of (soluble) groups for which this type of chain conditions are imposed to the set of all subgroups $(\max -\infty$ and $\min -\infty)$ or to set of all subnormal subgroups $(\max -\infty s n$ and $\min -\infty s n)$ was investigated by Paek [5], [6]. Moreover, he also studied the locally nilpotent groups with these conditions for normal subgroups $(\max -\infty n$ and min- $\infty n)$ [7], [8]. Recently, de Giovanni, Paek, Robinson and the author [2] have examined the structure of groups which satisfy max- $\infty$ n and min- $\infty$ n given characterizations of both properties in the general case. Moreover, detailed information is obtained about soluble groups with these chain conditions.

In the last section in [5] Paek shows that a subsoluble group (i.e., a group with an ascending series of subnormal subgroups whose factors are abelian) satisfying $\max -\infty s n$ (min- $\infty s n$, respectively) is soluble. Recall that a group $G$ is said to be generalized radical if it has an ascending series whose factors either are locally nilpotent or finite. As there exist locally nilpotent groups with trivial Baer radical (see, [9, pp. 24-26]), we have that the class of radical groups properly contains that of subsoluble groups. It is well known that a generalized radical group satisfying max-sn (min-sn, respectively) is polycyclic-by-finite (Chernikov, respectively) (see $[9,5.46]$ ). The aim of this short article is to prove the following extension of the latter and of the quoted result of Paek.

Theorem. Let $G$ be a generalized radical group which satisfies the condition $\max -\infty$ sn (the condition min- $\infty$ sn, respectively). Then $G$ is soluble-by-finite.

Most of our notation is standard, and can for instance be found in [9].

\section{Proof of Theorem}

In order to prove the theorem we need some preliminary results. Recall that a just infinite group is an infinite group all of whose proper quotients are finite.

Lemma 1. Let $G$ be any group. Then the following hold:

(i) If $G$ satisfies $\max -\infty n$ but not $\max -n$, then $G$ contains an infinite abelian normal subgroup $R$ such that $G / R$ has $\max -n$;

(ii) If $G$ satisfies min- $\infty$ n but not min- $n$, then $G$ contains a normal subgroup $N$ with $\min -G$ such that $G / N$ is just infinite.

Proof. See [2, 2.1 and 3.1].

Corollary 2. Let $G$ be a periodic hyper-(abelian or finite) group with min- $\infty$ n. Then $G$ satisfies min-n. 
Proof. Assume by contradiction that $G$ does not satisfy min- $n$. It follows by Lemma 1 that $G$ contains a normal subgroup $N$ such that $G / N$ is just infinite. Put $\bar{G}=G / N$, and let $\bar{A}$ be a non-trivial normal abelian subgroup of $\bar{G}$. Clearly, $\bar{A} \leq C_{\bar{G}}\left(\langle\bar{a}\rangle^{\bar{G}}\right)$ for each $\bar{a} \in \bar{A} \backslash\{1\}$. Therefore, $\bar{G} / C_{\bar{G}}\left(\langle\bar{a}\rangle^{\bar{G}}\right)$ is finite and hence $\bar{G}$ has max. Thus $\bar{G}$ is finite, which is a contradiction.

Our next result can also be deduced from the structure of locally nilpotent groups with max- $\infty$ ( $\min -\infty n$, respectively) obtained by Paek [7], [8]. But, for convenience of the reader, here we prefer to give an easy direct proof.

Lemma 3. Let $G$ be a locally nilpotent group satisfying $\max -\infty n(\min -\infty n$, respectively). Then $G$ is hypercentral.

Proof. First suppose that $G$ satisfies max- $\infty$ n. Clearly, if all normal non-trivial subgroups of $G$ are infinite, then by hypothesis $G$ has max- $n$ and hence it is nilpotent (see $[9,5.37]$ ). Therefore we can assume that $G$ contains a finite nontrivial normal subgroup and so even a minimal normal subgroup $M$. It follows that $M \leq Z(G)$ (see [9, Corollary of 5.27]). In particular, $Z(G) \neq\{1\}$. Thus $G$ is hypercentral since the class max- $\infty$ is closed with respect to homomorphic images.

Now let $G$ be a non-nilpotent group satisfying min- $\infty n$. Clearly $G$ is not just infinite, since otherwise it satisfies $\max -n$ and hence it is nilpotent. Let $N$ be a non-trivial normal subgroup of $G$ such that $G / N$ is infinite. By hypothesis $N$ contains a minimal $G$-invariant subgroup which is central in $G$. Therefore $G$ is hypercentral also in this case.

Lemma 4. Let $G$ be a generalized radical group satisfying max- $\infty$ sn (min$\infty$ sn, respectively). Then $G$ is hyper-(abelian or finite).

Proof. By Lemma 3 it is enough to prove that $G$ is hyper-(locally nilpotent or finite). Let $H$ be the Hirsch-Plotkin radical of $G$. Clearly we may assume that $H=\{1\}$ and hence $G$ has no non-trivial ascendant locally nilpotent subgroups. It follows that $G$ contains a non-trivial ascendant finite subgroup $F$. If $M$ is a subnormal non-abelian simple subgroup of $F$, then $M$ is also subnormal in $G$ by Corollary of 5.45 in [9]. Therefore the normal closure $M^{G}$ is finite since the direct product of infinitely many groups has neither max- $\infty$ n nor min- $\infty n$ (see $[9,5.44])$. It follows that $G$ contains a non-trivial normal subgroup which is either locally nilpotent or finite. Thus $G$ is hyper-(locally nilpotent or finite) as required.

Now we are in position to prove our theorem. Note that the argument used in the proof will show in particular that a periodic generalized radical group satisfying max- $\infty$ sn (min- $\infty s n$, respectively) is a Chernikov group.

Theorem 5. Let $G$ be a generalized radical group which satisfies the condition $\max -\infty$ sn (the condition min- $\infty$ sn, respectively). Then $G$ is soluble-by-finite. 
Proof. By Lemma 4, $G$ is hyper-(abelian or finite). First suppose that $G$ contains a non-periodic abelian normal subgroup $A$. Clearly, if $G$ satisfies max$\infty s n$, then $G / A$ has surely max-sn and hence it is polycyclic-by-finite. On the other hand, if $G$ has min- $\infty$ sn, then $G / A$ must be finite since otherwise $A$ satisfies min. Thus in either case $G$ is soluble-by-finite.

Now suppose that $G$ is periodic. We will prove that $G$ is a Chernikov group. First assume that $G$ satisfies $\max -\infty s n$. Clearly, if $G$ has $\max -n$, it is poly-(abelian or finite) and so even poly-(quasicyclic or finite) since an infinite periodic abelian group with max- $\infty$ is quasicyclic-by-finite (see $[6,3.1]$ ). It follows that $G$ is a Chernikov group. If $G$ does not satisfy max-n, Lemma 1 yields that $G$ contains an infinite abelian normal subgroup $R$ such that $G / R$ has max- $n$, and $G$ is again a Chernikov group by the previous argument. Now suppose that $G$ has min- $\infty s n$. Then $G$ satisfies min- $n$ by Corollary 2 . Let $B$ be a non-trivial normal abelian subgroup of $G$. Clearly, if $G / B$ is infinite, then $B$ has min and the same conclusion holds also when $G / B$ is finite by a well known theorem of Wilson (see $[9,5.21])$. It follows that $B$ contains a non-trivial finite $G$-invariant subgroup (see [9, Corollary 2 of 5.23$]$ ). Therefore $G$ is hyperfinite and hence a Chernikov group (see [9, Corollary 2 of 5.21]).

Finally, let $T$ be the largest periodic normal subgroup of $G$. By the previous paragraphes $T$ is a Chernikov group and $G / T$ is soluble-by-finite. It follows that $G$ is soluble-by-finite since a finite-by-abelian group is nilpotent-by-finite by a result of P. Hall (see [9, 4.25]).

\section{References}

[1] R. Baer, Polyminimaxgruppen, Math. Ann. 175 (1968), 1-43.

[2] F. de Giovanni, D. H. Paek, D. J. S. Robinson, and A. Russo, The maximal and minimal conditions for normal subgroups of infinite order or index, Comm. Algebra 33 (2005), no. 1, 183-199.

[3] L. A. Kurdachenko, Groups that satisfy weak minimality and maximality conditions for normal subgroups, Sibirsk. Mat. Zh. 20 (1979), no. 5, 1068-1076, 1167.

[4] _ Groups satisfying weak minimality and maximality conditions for subnormal subgroups, Mat. Zametki 29 (1981), no. 1, 19-30, 154.

[5] D. H. Paek, Chain conditions for subnormal subgroups of infinite order or index, Comm. Algebra 29 (2001), no. 7, 3069-3081.

[6] _ Chain conditions for subgroups of infinite order or index, J. Algebra 249 (2002), no. 2, 291-305.

[7] _ Locally nilpotent groups with the maximal condition on infinite normal subgroups, Bull. Korean Math. Soc. 41 (2004), no. 3, 465-472.

8] _ Locally nilpotent groups with the minimal condition on normal subgroups of infinite index, Bull. Korean Math. Soc. 41 (2004), no. 4, 779-783.

[9] D. J. S. Robinson, Finiteness Conditions and Generalized Soluble Groups, SpringerVerlag, New York-Berlin, 1972.

[10] - On the homology of hypercentral groups, Arch. Math. (Basel) 32 (1979), no. 3, $223-226$.

[11] D. I. Zaičev, Groups satisfying a weak minimal condition, Soviet Math. Dokl. 9 (1968), 194-197. 
ON GROUPS SATISFYING THE MAXIMAL AND THE MINIMAL CONDITIONS 691

[12] $\ldots$, On groups which satisfy a weak minimal condition, Math. USSR Sb. 7 (1969), $315-322$.

Dipartimento di Matematica

SEconda Università di NAPOLI

Via Vivaldi 43, 81100 Caserta, Italy

E-mail address: alessio.russo@unina2.it 